\title{
Axiomatizing the Harsanyi Solution, the Symmetric Egalitarian Solution, and the Consistent Solution for NTU-Games
}

Citation for published version (APA):

de Clippel, G., Peters, H. J. M., \& Zank, H. (2004). Axiomatizing the Harsanyi Solution, the Symmetric Egalitarian Solution, and the Consistent Solution for NTU-Games. International Journal of Game Theory, 33, 145-158. https://doi.org/10.1007/s001820400193

Document status and date:

Published: 01/01/2004

DOI:

$10.1007 / \mathrm{s} 001820400193$

Document Version:

Publisher's PDF, also known as Version of record

\section{Please check the document version of this publication:}

- A submitted manuscript is the version of the article upon submission and before peer-review. There can be important differences between the submitted version and the official published version of record.

People interested in the research are advised to contact the author for the final version of the publication, or visit the DOI to the publisher's website.

- The final author version and the galley proof are versions of the publication after peer review.

- The final published version features the final layout of the paper including the volume, issue and page numbers.

Link to publication

\footnotetext{
General rights rights.

- You may freely distribute the URL identifying the publication in the public portal. please follow below link for the End User Agreement:

www.umlib.nl/taverne-license

Take down policy

If you believe that this document breaches copyright please contact us at:

repository@maastrichtuniversity.nl

providing details and we will investigate your claim.
}

Copyright and moral rights for the publications made accessible in the public portal are retained by the authors and/or other copyright owners and it is a condition of accessing publications that users recognise and abide by the legal requirements associated with these

- Users may download and print one copy of any publication from the public portal for the purpose of private study or research.

- You may not further distribute the material or use it for any profit-making activity or commercial gain

If the publication is distributed under the terms of Article $25 \mathrm{fa}$ of the Dutch Copyright Act, indicated by the "Taverne" license above, 


\title{
Axiomatizing the Harsanyi solution, the symmetric egalitarian solution and the consistent solution for NTU-games*
}

\author{
Geoffroy de Clippel ${ }^{\dagger}$, Hans Peters ${ }^{\ddagger}$ and Horst Zank Z $^{£}$ \\ ${ }^{\dagger}$ Chargé de Recherches FNRS; CORE, Université catholique de Louvain, Belgium and \\ Department of Economics, Brown University, USA (e-mail: declippel@brown.edu.) \\ ${ }^{\ddagger}$ Department of Quantitative Economics, University of Maastricht, P.O. Box 616, 6200 MD \\ Maastricht, The Netherlands (e-mail: h.peters@ke.unimaas.nl.) \\ $\S$ School of Economic Studies, The University of Manchester, Oxford Road, Manchester, \\ M13 9PL, UK (e-mail: horst.zank@man.ac.uk.)
}

Revised October 2004

\begin{abstract}
The validity of the axiomatization of the Harsanyi solution for NTU-games by Hart (1985) is shown to depend on the regularity conditions imposed on games. Following this observation, we propose two related axiomatic characterizations, one of the symmetric egalitarian solution (Kalai and Samet, 1985) and one of the consistent solution (Maschler and Owen, 1992). The three axiomatic results are studied, evaluated and compared in detail.
\end{abstract}

JEL Classification: C71.

Key words: Nontransferable utility games, consistent solution, Harsanyi solution, symmetric egalitarain solution.

\section{Introduction}

Various solutions expressing some concern for fairness have been proposed for cooperative games in coalitional form with nontransferable utility (NTU). The main ones are the Harsanyi solution (cf. Harsanyi, 1963), the Shapley NTU solution (cf. Shapley, 1969), the symmetric egalitarian solution (cf. Kalai and Samet, 1985), and the consistent solution ${ }^{1}$ (cf. Maschler and Owen,

\footnotetext{
${ }^{*}$ We thank an anonymous referee and an associate editor for their helpful comments. Geoffroy de Clippel also thanks Professors Sergiu Hart, Jean-François Mertens and Enrico Minelli. Horst Zank thanks the Dutch Science Foundation NWO and the British Council for support under the UK-Netherlands Partnership Programme in Science (PPS 706). The usual disclaimer applies.

${ }^{1}$ Often the word 'value' is used instead of 'solution'. See Section 3 for the distinction between these concepts as adopted in this paper.
} 
1992). For brevity, we will call these solutions the H-solution, the S-solution, the KS-solution, and the MO-solution, respectively. All four solutions coincide with the Shapley (1953) value on the class of TU-games and all, except the KS-solution, coincide with the Nash bargaining solution (cf. Nash, 1950) on the class of pure bargaining problems. The KS-solution coincides with the symmetric proportional solution (cf. Kalai, 1977), also called egalitarian solution, on the class of pure bargaining problems. The S-solution has been axiomatized by Aumann (1985); the H-solution by Hart (1985); and the KS-solution by Kalai and Samet (1985).

The objective of the present paper is to provide new axiomatic characterizations of both the consistent and the symmetric egalitarian solutions. Our starting point is the axiomatization of the H-solution by Hart (1985). We show that its validity depends crucially on the regularity conditions that are imposed on feasible sets. In particular, we consider the following three domains:

1. games for which the feasible set of each coalition is positively smooth,

2. games for which only the feasible set of the grand coalition is required to be positively smooth,

3. games for which no smoothness assumption is made.

Here, 'positively smooth' means that there is a unique supporting hyperplane at each boundary point, with a normal vector that has all coordinates positive.

Hart's axioms characterize the H-solution on the second domain, but are indeterminate on the first one and incompatible on the third one. This can be seen as follows. First, the KS-solution is the only solution that satisfies all the axioms proposed by Hart, except scale covariance, on the third domain (see Proposition 2 hereafter). This provides an alternative axiomatization of the KS-solution. Second, the MO-solution satisfies all of Hart's axioms on the first domain, and is characterized on this domain by a natural strengthening of these axioms (see Proposition 3). The latter result is one of the first axiomatizations of the MO-solution for general NTU-games (with convex feasible sets). Hart (2003) presents another axiomatization (on a slightly different class of games) by means of a marginality axiom. Earlier papers already suggested alternative justifications. Maschler and Owen (1989) characterized the MOsolution on the class of hyperplane games. Hart and Mas-Colell (1996) proposed a non-cooperative procedure supporting the MO-solution.

In order to complete the picture, we refer the reader to Hart (1985) for a comparison between the S-solution and the H-solution through their axiomatizations. Our results are closer to Hart's axiomatization of the H-solution (working with payoff configurations) than to Aumann's axiomatization of the S-solution (working only with allocations for the grand coalition).

The paper is organized as follows. In the first three sections we introduce notations and definitions. In particular, we recall the definitions of the relevant solutions in Section 3. In Section 4 we review the main theorem of Hart (1985) and discuss this result by focusing on the importance of the regularity conditions imposed on games. The axiomatic characterizations of the KSsolution and the MO-solution are presented in Section 5. In Section 6 we reformulate the three axiomatic results in order to improve our understanding of the specific role of each axiom and the close dependence on the domains of 
definition. In Section 7 we provide examples to show logical independence of the axioms used in the characterizations. Proofs are given in Section 8.

\section{Notations}

Let $n$ be a positive integer, let $N:=\{1, \ldots, n\}$ be the set of players, and let $P(N)$ be the set of coalitions, that is, the set of nonempty subsets of $N$. The cardinality of a coalition $S$ is denoted by $s$. Elements of the Cartesian product $\prod_{S \in P(N)} \mathbb{R}^{S}$ are called payoff configurations, as they will represent profiles of payoff allocations that are contingent on the coalition that forms. For each coalition $S$ and pair of vectors $(\lambda, x)$ in $\mathbb{R}^{S} \times \mathbb{R}^{S}, \lambda x \in \mathbb{R}^{S}$ denotes the vector $\left(\lambda_{i} x_{i}\right)_{i \in S}$. This expression should be distinguished from the inner product $\lambda \cdot x:=\sum_{i \in S} \lambda_{i} x_{i}$. By $\Delta_{+}^{S}$ (resp. $\Delta_{++}^{S}$ ) we denote the set of vectors in $\mathbb{R}^{S}$ with nonnegative (resp. positive) components that sum up to 1 . Let $A$ be a convex subset of $\mathbb{R}^{S}$. Then the Pareto-frontier of $A$ (denoted by $\partial A$ ) is defined as follows:

$$
\partial A:=\left\{a \in A \mid \neg\left[\left(\exists a^{\prime} \in A\right): \forall i \in S, a_{i}^{\prime}>a_{i}\right]\right\} .
$$

The set $A \subseteq \mathbb{R}^{S}$ is said to be positively smooth if it admits a unique supporting hyperplane with a normal vector in $\Delta_{++}^{S}$ at each point of its Pareto-frontier.

\section{Games}

A game (with nontransferable utility, NTU) is a function that assigns a nonempty, closed, comprehensive, convex and proper subset of $\mathbb{R}^{S}$ to each coalition $S$. (A set $A \subseteq \mathbb{R}^{S}$ is comprehensive if for all $x \in A$ and $y \in \mathbb{R}^{S}$ with $y \leq x$ we have $y \in A$.) The class of all games is denoted by $\mathscr{G}$. A game $V$ is partially positively smooth if the set $V(N)$ is positively smooth. The class of all partially positively smooth games is denoted $\mathscr{G}_{P S m}^{\prime}$. Both Aumann (1985) and Hart (1985) essentially work with solutions defined on $\mathscr{G}_{P S m}^{\prime}$. A game $V$ is positively smooth if the set $V(S)$ is positively smooth for each coalition $S$. The class of all positively smooth games is denoted $\mathscr{G}_{P S m}$. Positive smoothness imposes the same regularity condition on the feasible set of each coalition. A similar class of games is considered in Hart and Mas-Colell (1996) who, additionally, impose a monotonicity condition. Obviously, $\mathscr{G}_{P S m} \subseteq \mathscr{G}_{P S m}^{\prime} \subseteq \mathscr{G}$. Operations on games are defined from operations on sets by applying these coalitionwise. Thus, for $V, W \in \mathscr{G}, \quad S \in P(N)$, and $\lambda \in \mathbb{R}_{++}^{N}$, we have: $(V+W)(S):=V(S)+W(S) \quad\left(:=\left\{z \in \mathbb{R}^{S} \mid \exists x \in V(S), y \in W(S): z=x+y\right\}\right) ;$ $(\partial V)(S):=\partial(V(S)) ;(\lambda V)(S):=\left\{y \in \mathbb{R}^{S} \mid \exists x \in V(S) \forall i \in S: y_{i}=\lambda_{i} x_{i}\right\}$. Similarly, $V \subseteq W$ means that $V(S) \subseteq W(S)$ for each $S \in P(N)$.

A $T \bar{U}$-game (transferable utility game) is a function $v: P(N) \rightarrow \mathbb{R}$. A game $V$ is equivalent to the $T U$-game $v$ if $V(S)=\left\{x \in \mathbb{R}^{S} \mid \sum_{i \in S} x_{i} \leq v(S)\right\}$ for each coalition $S$.

A hyperplane game $V$ is a game for which there exists a pair $(\lambda, v) \in\left(\prod_{S \in P(N)} \Delta_{++}^{S}\right) \times \mathbb{R}^{P(N)}$, where $\lambda=\left(\lambda_{S}\right)_{S \in P(N)}$, such that $V(S)=$ $\left\{x \in \mathbb{R}^{S} \mid \lambda_{S} \cdot x \leq v(S)\right\}$ for each coalition $S$. In that case, the pair $(\lambda, v)$ is uniquely determined. Every game that is equivalent to a TU-game is a hyperplane game. Every hyperplane game is positively smooth. 


\section{Values and solutions}

Let $G$ be a class of games. A value on $G$ is a function $\sigma$ that assigns a (unique) payoff configuration $\left(\sigma_{S}(V)\right)_{S \in P(N)}$ to each game belonging to $G$. The vector $\sigma_{S}(V) \in \mathbb{R}^{S}$ is called the value of $V$ for $S$, for each game $V$ in $G$ and each coalition $S$. A solution on $G$ is a function $\Sigma$ that assigns a (possibly empty) set of payoff configurations to each game belonging to $G$.

We now define three solutions on $\mathscr{G}$ : the KS-solution (the symmetric egalitarian solution of Kalai and Samet, 1985), the H-solution (the solution proposed by Harsanyi, 1963), and the MO-solution (the consistent solution of Maschler and Owen, 1992). To this end, we fix $V \in \mathscr{G}$.

The $K S$-value of $V$, denoted by $\sigma^{K S}(V)$, is the unique payoff configuration $\sigma \in \partial V$ for which there exists a vector $\xi \in \mathbb{R}^{P(N)}$ such that the $i$-th component of $\sigma_{S}$, denoted by $\sigma_{S, i}$, equals $\sum_{T \in P(S): i \in T} \xi_{T}$ for each $S \in P(N)$ and each $i \in S .^{2}$ It can be verified that $\sigma^{K S}$ is well defined. The $K S$-solution of $V$, denoted $\Sigma^{K S}(V)$, is simply the singleton containing $\sigma^{K S}(V)$. The KS-value can be understood as expressing a combination of efficiency $\left(\sigma^{K S}(V) \in \partial V\right)$ and equity (the real number $\xi_{T}$ being understood as a dividend distributed by coalition $T$ to each of its members).

The $H$-solution of $V$, denoted by $\Sigma^{H}(V)$, is the set of payoff configurations $\sigma \in \partial V$ for which there exists a pair $(\lambda, \xi) \in \Delta_{++}^{N} \times \mathbb{R}^{P(N)}$ such that $\lambda \cdot \sigma_{N}=\max _{y \in V(N)} \lambda \cdot y$ and $\lambda_{i} \sigma_{S, i}=\sum_{T \in P(S): i \in T} \xi_{T}$ for each $i \in S$ and each $S \in P(N)$. Each element $\sigma$ of the H-solution of $V$ combines an objective of efficiency $(\sigma \in \partial V)$, of utilitarianism in weighted utilities $\left(\lambda \cdot \sigma_{N}=\right.$ $\max _{y \in V(N)} \lambda \cdot y$ ), and of equity in weighted utilities (the real number $\xi_{T}$ being understood as a dividend in weighted utility distributed by coalition $T$ to each of its members), for some vector $\lambda$ of positive weights. The endogeneous determination of the weights in order to obtain the combination of different objectives is similar to the procedure proposed by Shapley (1969) in order to extend TU-solution concepts to NTU-games.

Maschler and Owen (1989) defined a value on the class of hyperplane games, inspired by the idea of marginal contributions underlying the Shapley value (cf. Shapley, 1953). Assume first that $V$ is a hyperplane game and let $S$ be a coalition. For each permutation $\pi$ of $S$, we define a marginal worth vector $M W(V, \pi) \in \mathbb{R}^{S}$ as follows:

$$
M W_{\pi(i)}(V, \pi):= \begin{cases}\max \left\{x_{\pi(i)} \mid x \in V(\{\pi(i)\})\right\} & \text { if } i=1 \\ \max \left\{x_{\pi(i)} \mid x \in V(\{\pi(1), \ldots, \pi(i)\})\right. & \\ \left.\wedge(\forall j \in\{1, \ldots, i-1\}): x_{\pi(j)}=M W_{\pi(j)}(V, \pi)\right\} & \text { if } i \geq 2\end{cases}
$$

for each $i \in\{1, \ldots, s\}$. The real number $M W_{\pi(i)}(V, \pi)$ determines the maximal payoff that player $\pi(i)$ can get if he is the $i^{\text {th }}$ player to enter the cooperation room, after players $\pi(1), \ldots, \pi(i-1)$ successively entered the room and were paid according to $M W(V, \pi)$. Then, the value of $V$ for $S$ is the vector of expected payoffs if all the permutations of the players in $S$ are equally likely, that is:

\footnotetext{
${ }^{2}$ Observe that, in accordance with the usual vector notation, we use the symbol $\sigma$ for values as well as for payoff configurations.
} 


$$
\sigma_{S}^{*}(V):=\frac{\sum_{\pi \in \Pi(S)} M W(V, \pi)}{s !},
$$

where $\Pi(S)$ denotes the set of all permutations of the players in $S .^{3}$

Maschler and Owen (1992) extended this value to a solution that is welldefined on $\mathscr{G}$. Consider an arbitrary game $V$ in $\mathscr{G}$. The consistent solution or $M O$-solution of $V$ (denoted by $\Sigma^{M O}(V)$ ) is the set of payoff configurations $\sigma \in \partial V$ for which there exists a $\lambda \in \prod_{S \in P(N)} \Delta_{+++}^{S}$ such that $\lambda_{S}$ is orthogonal to $V(S)$ at $\sigma_{S}$ for each $S \in P(N)$, and $\sigma=\sigma^{*}\left(V^{\lambda}\right)$, where $V^{\lambda}$ is the hyperplane game that is characterized by the pair $\left(\lambda,\left(\max _{y \in V(S)} \lambda_{S} \cdot y\right)_{S \in P(N)}\right)$. Each element $\sigma$ of $\Sigma^{M O}(V)$ (if any) thus specifies a vector of optimal allocations (one for each coalition) that coincides with the value (as defined above) of some hyperplane game that supports $V$ at $\sigma$.

\section{Hart's axiomatization of the Harsanyi solution}

Hart (1985) uses the following axioms in his characterization of the Harsanyi solution. We formulate these axioms for a solution $\Sigma$ defined on a class $G$ of games.

Axiom 1 (Efficiency, EFF) $\Sigma(V) \subseteq \partial V$ for each game $V \in G$.

Axiom 2 (Scale Covariance, SC) $\Sigma(\lambda V)=\lambda \Sigma(V)$ for each game $V \in G$ and each $\lambda \in \mathbb{R}_{++}^{N}$ such that $\lambda V \in G$.

Axiom 3 (Independence of Irrelevant Alternatives, IIA) $\Sigma(W) \cap V \subseteq \Sigma(V)$ for all games $V, W \in G$ such that $V \subseteq W$.

Axiom 4 (Conditional Super-Additivity, CSA) $[\Sigma(V)+\Sigma(W)] \cap \partial U \subseteq \Sigma(U)$ for all games $U, V, W \in G$ such that $U=V+W$.

Axiom 5 (Unanimity Games, $\mathrm{UG}$ ) Let $S$ be a coalition and let $c$ be a real number. The unanimity game $U_{c}^{S}$ is defined as follows: $U_{c}^{S}(T):=\left\{x \in \mathbb{R}^{T} \mid \sum_{i \in T} x_{i} \leq c\right\}$ for each coalition $T$ such that $S \subseteq T$, and $U_{c}^{S}(T):=\left\{x \in \mathbb{R}^{T} \mid \sum_{i \in T} x_{i} \leq 0\right\}$ for each other coalition $T$. If $U_{c}^{S^{c}} \in G$, then $\Sigma\left(U_{c}^{S}\right)=\{\sigma\}$, where $\sigma_{T}:=\frac{c}{s} 1_{S}$ for each coalition $T$ such that $S \subseteq T$, and $\sigma_{T}:=0$ for each other coalition $T{ }^{4}$

Axiom 6 (Zero-Inessential Games, ZIG) $0 \in \Sigma(V)$ for each game $V \in G$ such that $0 \in \partial V$.

The interpretation of these axioms is standard, see also Hart (1985).

Proposition 1(Hart, 1985) The H-solution (restricted to $\mathscr{G}_{P S m}^{\prime}$ ) is the only solution on $\mathscr{G}_{P S m}^{\prime}$ that satisfies Axioms 1-6.

The validity of Proposition 1 depends crucially on the set of games over which solutions are assumed to be defined. Indeed, $\Sigma^{M O}$ as a solution defined on the smaller class $\mathscr{G}_{P S m}$ satisfies Axioms 1-6 as well: Proposition 3 below implies that $\Sigma^{M O}$ satisfies Axioms 1, 3, and 4; Axioms 2, 5, and 6 can be verified directly. On the other hand, Axioms 1-6 (even 2-5) are incompatible on the larger class $\mathscr{G}$, as the following example shows.

\footnotetext{
${ }^{3}$ If $V$ is equivalent to a TU-game $v$, then $\sigma_{S}^{*}(V)$ is the Shapley value of $v$ restricted to $S$.

${ }^{4} 1_{S} \in \mathbb{R}^{T}$ is the vector with coordinates 1 for each $i \in S$ and 0 for each $i \in T \backslash S$.
} 
Example 1. Assume that there exists a solution $\Sigma$ defined on $\mathscr{G}$ that satisfies Axioms 2-5. Let $\lambda:=(2,1, \ldots, 1) \in \mathbb{R}^{N}$ and let $V:=\lambda U_{n}^{N}$. By Axioms 2 (SC) and $5(\mathrm{UG}), \Sigma(V)=\{\sigma\}$ where $\sigma_{N}:=\lambda$ and $\sigma_{S}:=0$, for each $S \in P(N) \backslash\{N\}$. Let $W$ be the game defined as follows:

$$
W(S):=\left\{x \in \mathbb{R}^{S} \mid x \leq \sigma_{S}\right\}
$$

for each $S \in P(N)$. Observe that the game $W$ is nonsmooth. By Axiom 3 (IIA), $\sigma \in \Sigma(W)$. By Axioms 4 (CSA) and 5 (UG), $\sigma \in \Sigma\left(W+U_{0}^{N}\right)=$ $\Sigma\left(U_{n+1}^{N}\right)$. On the other hand, Axiom 5 (UG) implies that $\Sigma\left(U_{n+1}^{N}\right)=\left\{\sigma^{\prime}\right\}$, where $\sigma_{N}^{\prime}:=\frac{n+1}{n}(1, \ldots, 1)$ and $\sigma_{S}^{\prime}:=0$, for each $S \in P(N) \backslash\{N\}$. This is a contradiction.

In view of the apparent impact of the regularity conditions imposed on games, one may ask if it is natural to consider a domain such as $\mathscr{G}_{P S m}^{\prime}$ in which the grand coalition and the smaller coalitions are treated asymmetrically.

\section{Axiomatization of the KS-solution and the MO-solution}

Following the observations made in the previous section we propose two axiomatic characterizations, one of the KS-solution and one of the MO-solution, that are technically very close to Hart's axiomatization of the $\mathrm{H}$-solution. The domains in these characterizations treat all coalitions symmetrically.

In the preceding section we observed that Axioms 1-6 are incompatible for solutions defined on $\mathscr{G}$. If, however, Axiom 2 (SC) is dropped we obtain a characterization of the KS-solution on $\mathscr{G}$.

Proposition 2. The KS-solution is the only solution on $\mathscr{G}$ that satisfies Axioms 1 (EFF), 3 (IIA), 4 (CSA), $5(U G)$ and 6 (ZIG).

Of course, the KS-solution violates Axiom 2. It does satisfy a weaker version requiring covariance only with respect to common positive rescaling of the utilities. The KS-solution has been axiomatized in a different way by Kalai and Samet (1985).

We also observed that Axioms 1-6 do not determine a unique solution on $\mathscr{G}_{P S m}$. Indeed, both the H-solution and the MO-solution satisfy these axioms on this domain.

The following axiom can be seen as strengthening both Axioms 5 (UG) and 6 (ZIG). It allows us to exactly characterize the MO-solution, even without appealing explicitly to Axiom 2 (SC). For a game $V$, a coalition $S$ with $s \geq 2$, a player $i \in S$, and a vector $y \in \mathbb{R}^{S \backslash i}$, define $x^{i}(V(S), y) \in \mathbb{R}^{S}$ by

$$
x_{k}^{i}(V(S), y):= \begin{cases}y_{k} & \text { if } k \neq i \\ \max \left\{x_{i} \mid x \in V(S) \wedge(\forall j \in S \backslash i): x_{j}=y_{j}\right\} & \text { if } k=i\end{cases}
$$

provided the maximum exists..$^{5}$ Next, define the payoff configuration $\sigma^{D} \equiv \sigma^{D}(V)$ (the superscript stands for 'dictator') recursively by

\footnotetext{
${ }^{5}$ This is the case if $\left\{x \in V(S) \mid(\forall j \in S \backslash i): x_{j}=y_{j}\right\}$ is nonempty and bounded. Note that we write $S \backslash i$ instead of $S \backslash\{i\}$.
} 
a) for all $i \in N, \sigma_{\{i\}}^{D}:=\max \{x \mid x \in V(\{i\})\}$,

b) for all $S \in P(N)$ with $s \geq 2, \sigma_{S}^{D}:=\frac{1}{s} \sum_{i \in S} x^{i}\left(V(S), \sigma_{S \backslash i}\right)$.

The payoff configuration $\sigma^{D}$ is well-defined (and unique) whenever all the $x^{i}(\cdot, \cdot)$ in b) exist; this is easily seen to be the case, in particular, for hyperplane games and zero-inessential games.

Axiom 7 (Recursive Conditional Random Dictatorship, RCRD) For all $V \in G$, if $\sigma^{D}(V) \in \partial V$ then $\Sigma(V)=\left\{\sigma^{D}(V)\right\}$.

We adopt the view that the fairness of a potential agreement for the grand coalition depends on what is feasible for that coalition and on what is thought to happen if a smaller coalition $S$ would form instead of $N$. Let us call the allocation achieved by $S$ the threat from $S$. If we focus on credible threats, somewhat similar to the idea of subgame perfection in extensive form games, then it is natural to view a solution payoff configuration as a collection of such threats that would actually be carried out if smaller coalitions would form. This interpretation is consistent with the (first) interpretation proposed by Hart (1985, p. 1299). It is also consistent with the principle of 'subcoalitional perfectness' suggested by Hart and Mas-Colell (1996, p. 366). Axiom 7 is then justified as follows by induction on the size of the coalitions. Let $V$ be a game for which the vector $\sigma^{D}$ as specified above is well-defined. It is clear that $\sigma_{\{i\}}^{D}$ is the credible threat from player $i$, for each $i \in N$. Next, let $S$ be a coalition with at least two members. Suppose that we already proved that $\sigma_{T}^{D}$ is the threat that would actually be carried out should some coalition $T \nsubseteq S$ form. The payoff allocation $x^{i}\left(V(S), \sigma_{S \backslash i}^{D}\right)$ specifies the choice of player $i \in S$, assuming that he has the dictatorial power to choose for $S$, under the participation constraint of the other players in $S$, who have the outside option to form a coalition without him in which case they obtain $\sigma_{S \backslash i}^{D}$ (by the induction hypothesis). The vector $\sigma_{S}^{D}$ then represents the expected payoff allocation for the players in $S$ if each member has an equal chance of obtaining this dictatorial power. If it is Pareto optimal in $V(S)$, then it is the threat that $S$ would carry out should $S$ form. We may summarize our reasoning as follows. The random dictatorship procedure determines for each coalition the only possible fair payoff allocation when its outcome is efficient. ${ }^{6}$ Axiom 7 is justified by applying this principle recursively, hence its name 'Recursive Conditional Random Dictatorship'.

Proposition 3. The MO-solution (restricted to $\mathscr{G}_{P S m}$ ) is the only solution on $\mathscr{G}_{P S m}$ that satisfies Axioms 1 (EFF), 3 (IIA), 4 (CSA) and 7 (RCRD).

Both the H-solution and the KS-solution satisfy Axioms 1, 3 and 4 on $\mathscr{G}_{P S m}$. Hence they cannot satisfy Axiom 7 . Here is an explicit example.

Example 2. Let $V$ be the three-player hyperplane game characterized by the pair $(\lambda, v)$, where $\lambda_{\{1\}}=\lambda_{\{2\}}=\lambda_{\{3\}}=1, \lambda_{\{1,2\}}=\left(\frac{1}{5}, \frac{4}{5}\right), \lambda_{\{1,3\}}=\left(\frac{1}{2}, \frac{1}{2}\right), \lambda_{\{2,3\}}=$ $\left(\frac{1}{2}, \frac{1}{2}\right), \lambda_{\{1,2,3\}}=\left(\frac{1}{3}, \frac{1}{3}, \frac{1}{3}\right), v(S)=0$, if $S$ equals $\{1\},\{2\},\{3\},\{1,3\}$, or $\{2,3\}$,

\footnotetext{
${ }^{6}$ It is easy to check that the consistent solution satisfies this principle beyond the specific content of Axiom 7: For each $V \in \mathscr{G}$, each $\sigma \in \Sigma^{M O}(V)$ and each $S \in P(N)$ with $s \geq 2$, if $\frac{\sum_{i \in S} x^{i}\left(V(S), \sigma_{S \backslash i}\right)}{s} \in \partial V(S)$, then $\sigma_{S}=\frac{\sum_{i \in S} x^{i}\left(V(S), \sigma_{S \mid i}\right)}{s}$.
} 
$v(\{1,2\})=20$, and $v(\{1,2,3\})=100 / 3$. The game $V$ is a slight variation on Owen's (1972) banker game. The interpretation is as follows. Player 1 can obtain $\$ 100$ with the help of player 2. Players 1 and 2 can transfer money to each other without the help of player 3 (the banker), but in that case only according to the exchange rate $4: 1$. When the three players cooperate, any split of the $\$ 100$ created through the cooperation of players 1 and 2 is feasible, thanks to the banker. If a solution $\Sigma$ satisfies Axiom 7, then $\Sigma(V)=\{\sigma\}$ where $\sigma_{S}=0$ if $S$ equals $\{1\},\{2\},\{3\}, \sigma_{S}=(0,0)$ if $S$ equals $\{1,3\}$ or $\{2,3\}$, $\sigma_{\{1,2\}}=(50,12.5)$, and $\sigma_{\{1,2,3\}}=(50,37.5,12.5)$. On the other hand, $\Sigma^{H}(V)=\Sigma^{K S}(V)=\left\{\sigma^{\prime}\right\}$, where $\sigma_{S}^{\prime}=0$ if $S$ equals $\{1\},\{2\},\{3\}, \sigma_{S}^{\prime}=(0,0)$ if $S$ equals $\{1,3\}$ or $\{2,3\}, \sigma_{\{1,2\}}^{\prime}=(20,20)$, and $\sigma_{\{1,2,3\}}^{\prime}=(40,40,20)$.

\section{A reformulation of the three axiomatic results}

In this section we reformulate Propositions $1-3$ in order to further clarify the role of each axiom and the impact of the different domain restrictions.

We introduce new axioms, stated for a solution $\Sigma$ defined on a class $G$ of games.

Axiom 4' (Independence of Non-dominating Alternatives, INA) $\Sigma(V) \cap \partial W$ $\subseteq \Sigma(W)$ for all games $V, W \in G$ such that $V \subseteq W$.

Consider a payoff configuration $\sigma$ that belongs to the solution of a game $V$. If $W$ is larger than $V$ (which means that each coalition has at least as many cooperative opportunities in $W$ than in $V$ ), and if, at the same time, $\sigma_{S}$ is not Pareto-dominated in the game $W$ (for each coalition $S$ ), then $\sigma$ should belong to the solution of $W$ as well. This axiom is a dual version of Axiom 3 (IIA). It is directly inspired by axioms appearing in Thomson and Myerson (1980) and in Thomson (1981). See also Chang and Hwang (2003) for a similar axiom in the context of NTU-games. Axiom $4^{\prime}$ is implied by the conjunction of Axioms 3 (IIA), 4 (CSA) and 6 (ZIG) (see Lemma 8 in Section 8 for a proof).

Axiom $7^{\prime} \Sigma(V)=\left\{\sigma^{*}(V)\right\}$ for each hyperplane game $V \in G$.

Axiom $7^{\prime \prime} \Sigma(\lambda V)=\left\{\sigma^{*}(\lambda V)\right\}$, for each $\lambda \in \Delta_{++}^{N}$ and each game $V \in G$ that is equivalent to a TU-game.

Axiom $7^{\prime \prime \prime} \Sigma(V)=\left\{\sigma^{*}(V)\right\}$, for each game $V \in G$ that is equivalent to a TUgame.

Recall from Section 3 that $\sigma_{S}^{*}(V) \in \mathbb{R}^{S}$ is the mean of the marginal worth vectors, for coalition $S$. It coincides with the Shapley value of $v$ restricted to $S$ when $V$ is equivalent to a TU-game $v$. It coincides by definition with the $S$-component of the unique payoff configuration in the MO-solution when $V$ is a hyperplane game. Axioms $7^{\prime}, 7^{\prime \prime}$ and $7^{\prime \prime \prime}$ are increasingly weaker. Note also that $7^{\prime \prime}$ is implied by $7^{\prime \prime \prime}$ together with Scale Covariance (Axiom 2), see also part 4 of Lemma 1.

Proposition 1'. The $H$-solution (restricted to $\mathscr{G}_{P S m}^{\prime}$ ) is the only solution on $\mathscr{G}_{P S m}^{\prime}$ that satisfies Axioms 1 (EFF), 3 (IIA), 4' (INA) and 7".

Proposition 2'. The KS-solution is the only solution on $\mathscr{G}$ that satisfies Axioms 1 (EFF), 3 (IIA), $4^{\prime}$ (INA) and $7^{\prime \prime \prime}$. 
Proposition $3^{\prime}$. The $M O$-solution (restricted to $\mathscr{G}_{P S m}$ ) is the only solution on $\mathscr{G}_{P S m}$ that satisfies Axioms 1 (EFF), 3 (IIA), $4^{\prime}$ (INA) and 7'.

Summarizing, the axioms EFF, IIA and INA, are used in all three propositions. The Axioms $7^{\prime}, 7^{\prime \prime}$, and $7^{\prime \prime \prime}$ are increasingly weaker but this is counterbalanced by the increasing domains $\mathscr{G}_{P s m} \subseteq \mathscr{G}_{P s m}^{\prime} \subseteq \mathscr{G}$ in Propositions $3^{\prime}, 1^{\prime}$ and $2^{\prime}$, respectively.

Finally, we note that Proposition $3^{\prime}$ can be decomposed into two parts: $\Sigma^{M O}$ is the minimal (resp. maximal) solution that satisfies Axioms 3 and $7^{\prime}$ (resp. 1, 4', and $7^{\prime}$ ). See the Remark at the end of Section 8 for the details.

\section{Independence of the axioms}

In this section we provide examples to show that the axioms used in Propositions 4-5 are logically independent. Below we define a number of solutions over an arbitrary set $N$ of players. In the following table, the rows correspond with the axioms and the columns with the three propositions. The solutions in the cells of this table satisfy all axioms in the proposition of the associated column with the exception of the axiom in the associated row. Proofs are left to the reader.

\begin{tabular}{llll}
\hline \#: Axiom & Prop. 1 $\mathscr{G}_{P s m}^{\prime}$ & Prop. 2 $\mathscr{G}$ & Prop. 3 $\mathscr{G}_{P s m}$ \\
\hline 1: EFF & $\Sigma_{+0}^{H}$ & $\Sigma_{+0}^{K S}$ & $\Sigma_{1}$ \\
2: SC & $\Sigma^{K S}$ & & \\
3: IIA & $\Sigma_{0 h}^{H}$ & $\Sigma_{0 h}^{K S}$ & $\Sigma_{2}$ \\
4: CSA & $\Sigma_{\partial h}^{H}$ & $\Sigma_{\partial h}^{K S}$ & $\Sigma_{3}$ \\
5: UG & $\Sigma_{w}^{H}$ & $\Sigma_{w}^{K S}$ & \\
6: ZIG & $\Sigma^{H} \cap \Sigma^{S}$ & $\widehat{\Sigma}$ & \\
7: RCRD & & & $\Sigma^{K S}$ \\
\hline
\end{tabular}

(i) Let $G$ be a subset of games and $\Sigma$ a solution on $G$. The solution $\Sigma_{+0}$ on $G$ is defined as follows. If $V$ is not a hyperplane game and $0 \in V$, then $\Sigma_{+0}(V):=\Sigma(V) \cup\{0\}$. Otherwise, $\Sigma_{+0}(V):=\Sigma(V)$.

(ii) Let $G$ be a subset of games and $\Sigma$ a solution on $G$. The solution $\Sigma_{0 h}$ on $G$ is defined as follows. If $0 \in \partial V$, then $\Sigma_{0 h}(V):=\{0\}$. If $V$ is a hyperplane game, then $\Sigma_{0 h}(V):=\Sigma(V)$. Otherwise, $\Sigma_{0 h}(V):=\emptyset$.

(iii) Let $G$ be a subset of games and $\Sigma$ a solution on $G$. The solution $\Sigma_{\partial h}$ on $G$ is defined as follows. If $V$ is a hyperplane game, then $\Sigma_{\partial h}(V):=\Sigma(V)$. Otherwise, $\Sigma_{\partial h}(V):=\prod_{S \in P(N)} \partial V(S)$.

(iv) $\Sigma_{w}^{K S}$ is some weighted proportional solution on $\mathscr{G}$ (see Kalai and Samet, 1985). Similarly, $\Sigma_{w}^{H}$ is some weighted version of the Harsanyi solution on $\mathscr{G}_{P S m}^{\prime}$ (details can be filled in by the reader).

(v) Let $\Sigma^{S}$ denote the Shapley (1969) NTU solution as formulated by Hart (1985, Section 5) in terms of payoff configurations: for each $V \in \mathscr{G}$, $\sigma \in \Sigma^{S}(V)$ if and only if there exists $\lambda \in \Delta_{++}^{N}$ such that $\sum_{i \in S} \lambda_{i} v_{i} \leq$ $\sum_{i \in S} \lambda_{i} \sigma_{S, i}$ for each $S \in P(N)$ and $\sigma_{N}$ is the Shapley (1953) value of the TU-game $v^{\lambda}$ defined by $v^{\lambda}(S):=\sum_{i \in S} \lambda_{i} \sigma_{S, i}$ for each $S \in P(N)$. Hart (1985, Theorem B) proved that the Harsanyi-Shapley solution $\Sigma^{H} \cap \Sigma^{S}$ 
is the minimal solution on $\mathscr{G}_{P S m}^{\prime}$ to satisfy Axioms 1-5. The minimal solution on $\mathscr{G}$ to satisfy Axioms $1,3,4$, and 5 is the solution $\widehat{\Sigma}$ defined by $\widehat{\Sigma}(V):=\left\{\sigma \in \Sigma^{K S}(V) \mid \forall S \in P(N): \sum_{i \in S} \sigma_{S, i}=\max _{x \in V(S)} \Sigma_{i \in S} x_{i}\right\}$ for every $V \in \mathscr{G}$.

(vi) Let $\hat{V}$ be a positively smooth game satisfying the following conditions: 1) $\hat{V}(\{1,2\})$ is symmetric with $\{(4,0),(0,4),(3,3)\} \subset \partial \hat{V}(\{1,2\}) ; 2)$ $\max \{x \mid x \in \hat{V}(\{i\})\}=1$ for each $i \in\{1,2\} ; 3) \quad\left\{\left(x_{1}, x_{2}\right) \in \mathbb{R}^{\{1,2\}} \mid\right.$ $\left.\left(x_{1}, x_{2}, 0, \ldots, 0\right) \in V(S)\right\}=\hat{V}(\{1,2\})$ for each coalition $S$ such that $\{1,2\} \subseteq S$; 4) $0 \in \partial \hat{V}(S)$ for each coalition $S$ that does not contain $\{1,2\}$ and that is different from $\{1\}$ and $\{2\} . \hat{V}$ is essentially a symmetric bargaining problem between players 1 and 2, the other players (if any) being dummies. Let $\sigma$ be the payoff configuration defined as follows: $\sigma_{S}:=(3,3,0, \ldots, 0) \in \mathbb{R}^{S}$ for each coalition $S$ such that $\{1,2\} \subseteq S$ and $\sigma_{S}:=0 \in \mathbb{R}^{S}$ for each other coalition. Notice that $\sigma \in \Sigma^{M O}\left(\hat{V}^{\prime}\right)$ where $\hat{V}^{\prime}(S)=\hat{V}(S)$ for each coalition $S$ with at least two members and $\hat{V}^{\prime}(\{i\}):=\{x \in \mathbb{R} \mid x \leq 0\}$ for each $i \in N$. The solution $\Sigma_{1}$ on $\mathscr{G}_{P S m}$ is defined as follows. If $V \subseteq \hat{V}$ such that $\sigma \in V$, then $\Sigma_{1}(V):=\Sigma^{M O}(V) \cup\{\sigma\}$. Otherwise, $\Sigma_{1}(V):=\Sigma^{M O}(V)$.

(vii) Let $G$ be the class of games $V \in \mathscr{G}_{P S m}$ for which the payoff configuration $\sigma^{D}$ as specified before Axiom 7 is well-defined and $\sigma^{D} \in \partial V$. Then $\Sigma_{2}$ is defined as follows: $\Sigma_{2}(V):=\left\{\sigma^{D}\right\}$ if $V \in G$ and $\Sigma_{2}(V):=\emptyset$ if $V \in \mathscr{G}_{P S m} \backslash G$.

(viii) Let $V \in \mathscr{G}_{P S m}$. Then $\Sigma_{3}(V)$ is the set of payoff configurations $\sigma \in \partial V$ such that $x^{i}\left(V(S), \sigma_{S \backslash i}\right)$ exists for each $i \in S$ and $\sigma_{S} \geq \sum_{i \in S} x^{i}\left(V(S), \sigma_{S \backslash i}\right) / s$ for each coalition $S$ with at least two members.

\section{Proofs}

We first state some logical relations between the axioms.

Lemma 1. Let $G$ be any of the sets $\mathscr{G}, \mathscr{G}_{P S m}^{\prime}$, or $\mathscr{G}_{P S m}$, and let $\Sigma$ be a solution on G. Then:

1) $7 \Rightarrow 7^{\prime} \Rightarrow 7^{\prime \prime} \Rightarrow 7^{\prime \prime \prime} \Rightarrow 5$;

2) $3 \& 4 \& 6 \Rightarrow 4^{\prime}$;

3) $1 \& 4 \& 5 \Rightarrow 7^{\prime \prime \prime}$

4) $2 \& 7^{\prime \prime \prime} \Rightarrow 7^{\prime \prime}$.

Proof. The implication $7 \Rightarrow 7^{\prime}$ is a direct consequence of Lemma 4 in Subsection 8.3. The rest of part 1) as well as part 4) are easy to prove. Also part 3) is not difficult to prove: basically, it corresponds to a standard characterization of the Shapley value for TU-games. For part 2), let $V$ and $W$ be two games such that $V \subseteq W$, let $\sigma \in \Sigma(V) \cap \partial W$, and let $\lambda \in \prod_{S \in P(N)} \Delta_{+}^{S}$ be a (not necessarily unique) profile of normalized vectors such that $\lambda_{S}$ is orthogonal to $W(S)$ at $\sigma_{S}$. Let $W^{\prime}$ be the game defined by:

$$
W^{\prime}(S):=\left\{x \in \mathbb{R}^{S} \mid \lambda_{S} \cdot x \leq 0\right\}
$$

for each $S \in P(N)$. By Axiom 6 (ZIG), $0 \in \Sigma\left(W^{\prime}\right)$. By Axiom 4 (CSA), $\sigma \in \Sigma\left(W^{\lambda}\right)$, where $W^{\lambda}:=V+W^{\prime}$. By Axiom 3 (IIA), $\sigma \in \Sigma(W)$ since $W \subseteq W^{\lambda}$. This proves $4^{\prime}$ (INA). 


\subsection{Proof of Propositions 1 and $1^{\prime}$}

The proofs of Propositions 1 and $1^{\prime}$ are similar to the proof of the main theorem of Hart (1985). We nevertheless include the proofs, not only for completeness, but also because the slightly different exposition of the arguments will allow the reader to make comparisons with the proofs of Propositions $2,2^{\prime}, 3$ and $3^{\prime}$.

We refer the reader to Proposition 4.11 of Hart (1985) for the proof of the fact that the H-solution satisfies axioms $1,2,3,4,5$ and 6 on $\mathscr{G}_{P S m}^{\prime}$.

Lemma 2. Let the solution $\Sigma$ on $\mathscr{G}_{P S m}^{\prime}$ satisfy Axioms 1 (EFF), 3 (IIA), $4^{\prime}$ (INA) and $7^{\prime \prime}$. Then $\Sigma=\Sigma^{H}$.

Proof: Let $V \in \mathscr{G}_{P S m}^{\prime}$, let $\sigma \in \partial V$, let $\lambda \in \Delta_{++}^{N}$ be the normalized vector that is orthogonal to $V(N)$ at $\sigma_{N}$, let $V_{\sigma}^{\lambda}$ be the game defined by:

$$
V_{\sigma}^{\lambda}(S):=\left\{x \in \mathbb{R}^{S} \mid \sum_{i \in S} \lambda_{i} x_{i} \leq \sum_{i \in S} \lambda_{i} \sigma_{S, i}\right\}
$$

for each $S \in P(N)$, and let $W$ be the game defined by $W(N):=V(N)$ and

$$
W(S):=\left\{x \in \mathbb{R}^{S} \mid x \leq \sigma_{S}\right\}
$$

for each $S \in P(N) \backslash\{N\}$. We note that $W \subseteq V_{\sigma}^{\lambda}$, that $W \subseteq V$, that $\sigma \in \partial V_{\sigma}^{\lambda} \cap \partial W$, and that both $V_{\sigma}^{\lambda}$ and $W$ are partially positively smooth. Then:

$$
\begin{aligned}
\sigma \in \Sigma^{H}(V) & \Longleftrightarrow \sigma \in \Sigma^{H}\left(V_{\sigma}^{\lambda}\right)\left(\text { by definition of } \Sigma^{H}\right) \\
& \Longleftrightarrow \sigma \in \Sigma\left(V_{\sigma}^{\lambda}\right)\left(\text { both } \Sigma \text { and } \Sigma^{H} \text { satisfy Axiom } 7^{\prime \prime}\right) \\
& \Longleftrightarrow \sigma \in \Sigma(W)\left(\text { Axioms } 3 \text { and } 4^{\prime}\right) \\
& \Longleftrightarrow \sigma \in \Sigma(V)\left(\text { Axioms } 3 \text { and } 4^{\prime}\right) .
\end{aligned}
$$

This completes the proof since both $\Sigma$ and $\Sigma^{H}$ satisfy Axiom 1 (EFF).

Propositions 1 and $1^{\prime}$ follow from Lemmas 1 and 2 .

\subsection{Proof of Propositions 2 and $2^{\prime}$}

It is easy to check that the symmetric KS-solution satisfies Axioms 1, 3, 4, 5, 6 and $7^{\prime \prime \prime}$ on $\mathscr{G}$.

Lemma 3. Let the solution $\Sigma$ on $\mathscr{G}$ satisfy Axioms 1 (EFF), 3 (IIA), 4' (INA) and $7^{\prime \prime \prime}$. Then $\Sigma=\Sigma^{K S}$.

Proof: Let $V \in \mathscr{G}$, let $\sigma \in \partial V$, let $V_{\sigma}$ be the game defined by:

$$
V_{\sigma}(S):=\left\{x \in \mathbb{R}^{S} \mid \sum_{i \in S} x_{i} \leq \sum_{i \in S} \sigma_{S, i}\right\}
$$

for each $S \in P(N)$, and let $\widehat{W}$ be the game defined by:

$$
\widehat{W}(S):=\left\{x \in \mathbb{R}^{S} \mid x \leq \sigma_{S}\right\}
$$

for each $S \in P(N)$. We note that $\widehat{W} \subseteq V_{\sigma}$, that $\widehat{W} \subseteq V$, and that $\sigma \in \partial V_{\sigma} \cap \partial \widehat{W}$. We have: 


$$
\begin{aligned}
\sigma \in \Sigma^{K S}(V) & \left.\Longleftrightarrow \sigma \in \Sigma^{K S}\left(V_{\sigma}\right) \text { (by definition of } \Sigma^{K S}\right) \\
& \Longleftrightarrow \sigma \in \Sigma\left(V_{\sigma}\right)\left(\text { both } \Sigma \text { and } \Sigma^{K S} \text { satisfy Axiom } 7^{\prime \prime \prime}\right) \\
& \left.\Longleftrightarrow \sigma \in \Sigma(\widehat{W}) \text { (Axioms } 3 \text { and } 4^{\prime}\right) \\
& \left.\Longleftrightarrow \sigma \in \Sigma(V) \text { (Axioms } 3 \text { and } 4^{\prime}\right) .
\end{aligned}
$$

This completes the proof since both $\Sigma$ and $\Sigma^{K S}$ satisfy Axiom 1 (EFF).

Propositions 2 and $2^{\prime}$ now follow from Lemmas 1 and 3.

\subsection{Proof of Propositions 3 and $3^{\prime}$}

The next lemma directly follows from Maschler and Owen (1989, Lemma 1). Recall the definition of $\sigma^{D}(V)$ before Axiom 7.

Lemma 4. Let $V$ be a hyperplane game. Then $\sigma^{*}(V)=\sigma^{D}(V)$.

The following additional notations are useful in what follows. Let $V \in \mathscr{G}_{P S m}$ and let $\sigma \in \partial V$. Then $\lambda(\sigma, V) \in \prod_{S \in P(N)} \Delta_{++}^{S}$ denotes the profile of normalized vectors whose $S$-component is orthogonal to $V(S)$ at $\sigma_{S}$, for each $S \in P(N)$. Also, $V^{\lambda(\sigma, V)}$ denotes the hyperplane game that is characterized by the pair $\left(\lambda(\sigma, V),\left(\max _{y \in V(S)} \lambda_{S}(\sigma, V) \cdot y\right)_{S \in P(N)}\right)$.

Lemma 5. $\Sigma^{M O}$, as a solution defined on $\mathscr{G}_{P S m}$, satisfies Axiom 7 (RCRD).

Proof: Let $V \in \mathscr{G}_{P S m}$. Suppose that the payoff configuration $\sigma^{D} \equiv \sigma^{D}(V)$ as defined before Axiom 7 is well-defined and that $\sigma^{D} \in \partial V$. Write $\sigma$ instead of $\sigma^{D}$. Let $\sigma^{\prime} \in \Sigma^{M O}(V)$. We prove by induction on the size of $S$ that $\sigma_{S}^{\prime}=\sigma_{S}$ for each $S \in P(N)$. The property is clearly true for singletons. So, let $s \geq 2$, let $S$ be a coalition of size $s$ and assume that $\sigma_{S \backslash i}^{\prime}=\sigma_{S \backslash i}$ for each $i \in S$. Then, $\sigma_{S}^{\prime}=\frac{1}{s} \sum_{i \in S} x^{i}\left(V^{\lambda\left(\sigma^{\prime}, V\right)} \quad(S), \sigma_{S \backslash i}^{\prime}\right) \geq \frac{1}{s} \quad \sum_{i \in S} x^{i}\left(V(S), \sigma_{S \backslash i}^{\prime}\right)=\frac{1}{s} \sum_{i \in S} x^{i}(V(S)$, $\left.\sigma_{S \backslash i}\right)=\sigma_{S}$. The first equality follows from Lemma 4 . The last equality follows from the definition of $\sigma\left(=\sigma^{D}\right)$. As $\sigma_{S}^{\prime} \in V(S)$ and $\sigma_{S} \in \partial V(S)$, we have $\sigma_{S}^{\prime}=\sigma_{S}$. Hence, by induction it follows that $\sigma^{\prime}=\sigma$. So we have proved that if $\Sigma^{M O}(V) \neq \emptyset$, then $\Sigma^{M O}(V)=\{\sigma\}$. We now prove that $\sigma \in \Sigma^{M O}(V)$. This amounts to proving that $\sigma_{S}=\sigma_{S}^{*}\left(V^{\lambda(\sigma, V)}\right)$ for each coalition $S$. Once again we proceed by induction on the size of $S$. The property is clearly true for singletons. So, let $s \geq 2$, let $S$ be a coalition of size $s$ and assume that $\sigma_{S \backslash i}=\sigma_{S \backslash i}^{*}\left(V^{\lambda(\sigma, V)}\right)$ for each $i \in S$. As $\sigma_{S}=\sum_{i \in S} x^{i}\left(V(S), \sigma_{S \backslash i}\right) / s$ by definition of $\sigma$ and $\sigma_{S}^{*}\left(V^{\lambda(\sigma, V)}\right)=\sum_{i \in S} x^{i}\left(V^{\lambda(\sigma, V)}(S), \sigma_{S \backslash i}^{*}\left(V^{\lambda(\sigma, V)}\right)\right) / s$ by Lemma 4 , it is sufficient to prove that $x^{i}\left(V(S), \sigma_{S \backslash i}\right)=x^{i}\left(V^{i(\sigma, V)}(S), \sigma_{S \backslash i}\right)$ for each $i \in S$ or, equivalently, that $\max \left\{x_{i} \mid x \in V(S) \wedge(\forall j \in S \backslash i): x_{j}=\left(\sigma_{S \backslash i}\right)_{j}\right\}=\max \left\{x_{i} \mid\right.$ $\left.x \in V^{\lambda(\sigma, V)}(S) \wedge(\forall j \in S \backslash i): x_{j}=\left(\sigma_{S \backslash i}\right)_{j}\right\}$. First, notice that $\max \left\{x_{i} \mid x\right.$ $\left.\in V(S) \wedge(\forall j \in S \backslash i): x_{j}=\left(\sigma_{S \backslash i}\right)_{j}\right\} \leq \max \left\{x_{i} \mid x \in V^{\lambda(\sigma, V)}(S) \wedge(\forall j \in S \backslash i):\right.$ $\left.x_{j}=\left(\sigma_{S \backslash i}\right)_{j}\right\}$ for each $i \in S$, since $V(S) \subseteq V^{\lambda(\sigma, V)}(S)$. Second, if there exists some player $i \in S$ for which this inequality is strict, then $\lambda_{S}(\sigma, V) \cdot\left(\frac{1}{s} \sum_{i \in S} x_{i}\left(V(S), \sigma_{S \backslash i}\right)\right)<\lambda_{S}(\sigma, V) \cdot \sigma_{S}$, which contradicts the fact that $\sigma_{S}=\frac{1}{s} \sum_{i \in S} x_{i}\left(V(S), \sigma_{S \backslash i}\right)$. 
Lemma 6. (Conditional additivity of the MO-solution on the class of hyperplane games). Let $\lambda \in \prod_{S \in P(N)} \Delta_{++}^{S}$ be a profile of normalized vectors, let $v: P(N) \rightarrow \mathbb{R}$ and $w: P(N) \rightarrow \mathbb{R}$ be two functions, and let $V$ and $W$ be the two hyperplane games that are characterized by the pairs $(\lambda, v)$ and $(\lambda, w)$, respectively. Then $\sigma^{*}(V+W)=\sigma^{*}(V)+\sigma^{*}(W)$.

Proof: We prove that $\sigma_{S}^{*}(V+W)=\sigma_{S}^{*}(V)+\sigma_{S}^{*}(W)$ for each $S \in P(N)$, by induction on the cardinality of $S$. If $S$ is a singleton, then the result is obvious by definition of $\sigma^{*}$. Assume $s \geq 2$ and the statement holds for all coalitions of cardinality $s-1$. Notice that $V+W$ is the hyperplane game characterized by the pair $(\lambda, v+w)$. Let $i \in S$. We have:

$$
\max _{x \in(V+W)(S):\left(x_{j}\right)_{j \in S \backslash i}=\sigma_{S \backslash i}^{*}(V+W)} x_{i}=\frac{(v+w)(S)-\sum_{j \in S \backslash i} \lambda_{S, j}\left(\sigma_{S \backslash i}^{*}\right)_{j}(V+W)}{\lambda_{S, i}} .
$$

By the induction assumption, the right-hand side expression equals

$$
\frac{v(S)-\sum_{j \in S \backslash i} \lambda_{S, j}\left(\sigma_{S \backslash i}^{*}\right)_{j}(V)}{\lambda_{S, i}}+\frac{w(S)-\sum_{j \in S \backslash i} \lambda_{S, j}\left(\sigma_{S \backslash i}^{*}\right)_{j}(W)}{\lambda_{S, i}},
$$

which, in turn, equals

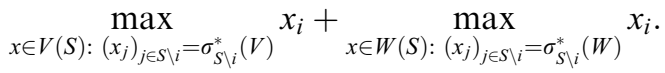

So, $x^{i}\left((V+W)(S), \sigma_{S \backslash i}^{*}(V+W)\right)=x^{i}\left(V(S), \sigma_{S \backslash i}^{*}(V)\right)+x^{i}\left(W(S), \sigma_{S \backslash i}^{*}(W)\right)$ for each $i \in S$. The proof is completed by using Lemma 4 .

Lemma 7. $\Sigma^{M O}$, as a solution defined on $\mathscr{G}_{P S m}$, satisfies Axiom 4 (CSA).

Proof: Let $V \in \mathscr{G}_{P S m}$ and $W \in \mathscr{G}_{P S m}$ be such that $U:=V+W \in \mathscr{G}_{P S m}$. Let $\sigma \in \Sigma^{M O}(V)$ and $\sigma^{\prime} \in \Sigma^{M O}(W)$ be such that $\sigma+\sigma^{\prime} \in \partial U$. Then $\lambda(\sigma, V)=\lambda\left(\sigma^{\prime}, W\right)=\lambda\left(\sigma+\sigma^{\prime}, U\right)$. For simplicity we denote this profile of vectors by $\lambda$. Then $U^{\lambda}=V^{\lambda}+W^{\lambda}$. So, by Lemma 6 , we have: $\sigma^{*}\left(U^{\lambda}\right)=$ $\sigma^{*}\left(V^{\lambda}\right)+\sigma^{*}\left(W^{\lambda}\right)=\sigma+\sigma^{\prime}$. This concludes the proof.

Lemma 8. Let the solution $\Sigma$ on $\mathscr{G}_{P S m}$ satisfy Axioms $1,3,4^{\prime}$ and $7^{\prime}$. Then $\Sigma=\Sigma^{M O}$.

Proof: Let $V \in \mathscr{G}_{P S m}$ and let $\sigma \in \partial V$. We may thus consider the profile of normalized vectors $\lambda(\sigma, V)$. Then:

$$
\begin{aligned}
\sigma \in \Sigma^{M O}(V) & \Longleftrightarrow \sigma \in \Sigma^{M O}\left(V^{\lambda(\sigma, V)}\right)\left(\text { by definition of } \Sigma^{M O}\right) \\
& \Longleftrightarrow \sigma \in \Sigma\left(V^{\lambda(\sigma, V)}\right)\left(\text { both } \Sigma \text { and } \Sigma^{M O} \text { satisfy Axiom } 7^{\prime}\right) \\
& \Longleftrightarrow \sigma \in \Sigma(V)\left(\text { Axioms } 3 \text { and } 4^{\prime}\right) .
\end{aligned}
$$

The proof is complete by using the fact that both $\Sigma$ and $\Sigma^{M O}$ satisfy Axiom 1 (EFF).

It is easy to verify that the MO-solution satisfies Axioms 1 (EFF), 3 (IIA), and 6 (ZIG) on $\mathscr{G}_{P S m}$. (Observe that ZIG is also implied by RCRD.) By Lemmas 5 and 7 it also satisfies Axioms 7 (RCRD) and 4 (CSA) on $\mathscr{G}_{P S m}$. 
Hence, by Lemma 1 it satisfies Axioms $4^{\prime}$ (INA) and $7^{\prime}$ on this class. Together with Lemma 8 this implies both Propositions 3 and $3^{\prime}$.

Remark. Looking at the proof of Lemma 8, we observe that Proposition $3^{\prime}$ can be decomposed into two parts: $\Sigma^{M O}$ is the minimal (resp. maximal) solution that satisfies Axioms 3 and $7^{\prime}$ (resp. 1, 4' and $7^{\prime}$ ). In fact, if the MO-solution were single-valued (which is not true in general), either of the two parts would be sufficient in order to obtain the exact characterization. This point is well illustrated by the papers of Nash (1950), who adopts the approach with Axiom 3 (IIA), and of Thomson (1981), who adopts the approach with Axiom 4' (INA), for their axiomatizations of the (singlevalued) Nash bargaining solution on the class of pure bargaining games. The same kind of decomposition does not necessarily apply to Propositions $1^{\prime}$ and $2^{\prime}$, as both Axioms 3 and $4^{\prime}$ are used in order to prove the two inclusions that are required to obtain the equalities in Lemmas 2 and 3 .

\section{References}

[1] Aumann RJ (1985) An Axiomatization of the Non-Transferable Utility Value. Econometrica 53:599-612

[2] Chang C, Hwang Y-A (2003) The Harsanyi-Shapley Solution and Independence of Irrelevant Expansions. International Journal of Game Theory. 32:253-271

[3] Harsanyi JC (1963) A Simplified Bargaining Model for the n-Person Cooperative Game. International Economic Review. 4:194-220

[4] Hart S (1985) An Axiomatization of Harsanyi's Nontransferable Utility Solution. Econometrica 53:1295-1313

[5] Hart S (2003) An Axiomatization of the Consistent Non-Transferable Utility Value. The Hebrew University of Jerusalem, Center for Rationality DP-337

[6] Hart S, Mas-Collel A (1996) Bargaining and Value. Econometrica 64:357-380

[7] Kalai E (1977) Proportional Solutions to Bargaining Situations: Interpersonal Utility Comparisons. Econometrica 45:1623-1630

[8] Kalai E, Samet D (1985) Monotonic Solutions to General Cooperative Games. Econometrica 53:307-327

[9] Maschler M, Owen G (1989) The Consistent Shapley Value for Hyperplane Games. International Journal of Game Theory 18:389-407

[10] Maschler M, Owen G (1992) The consistent shapley value for games without side payments. In: Selten R (ed) Rational Interaction, Springer-Verlag, New York, 5-12

[11] Nash JF (1950) The Bargaining Problem. Econometrica 18:155-162

[12] Owen G (1972) Values of Games without Side Payments. International Journal of Game Theory 1:95-109

[13] Shapley LS (1953) A Value for $n$-Person Games. In: Tucker AW, Kuhn HW, (eds)Contributions to the Theory of Games II, Princeton University Press, Princeton, NJ, 307-317

[14] Shapley LS (1969) Utility Comparisons and the Theory of Games. In: La Decision G, Guilbaud Th (ed) CNRS, Paris 251-263

[15] Thomson W (1981) Independence of Irrelevant Expansions. International Journal of Game Theory 10:107-114

[16] Thomson W, Myerson RB (1980) Monotonicity and Independence Axioms. International Journal of Game Theory. 9:37-49 
Copyright of International Journal of Game Theory is the property of Springer - Verlag New York, Inc. and its content may not be copied or emailed to multiple sites or posted to a listserv without the copyright holder's express written permission. However, users may print, download, or email articles for individual use. 
Copyright of International Journal of Game Theory is the property of Springer Science \& Business Media B.V.. The copyright in an individual article may be maintained by the author in certain cases. Content may not be copied or emailed to multiple sites or posted to a listserv without the copyright holder's express written permission. However, users may print, download, or email articles for individual use. 